\title{
Electrophysiological evaluation of visual loss in Müller cell sheen dystrophy
} Ulrich Kellner, Hannelore Kraus, Heinrich Heimann, Horst Helbig, Norbert Bornfeld,
Michael H Foerster

\begin{abstract}
Aims-To describe the clinical picture and electrophysiological findings in Müller cell sheen dystrophy, a recently reported retinal dystrophy.

Method-A basic ophthalmological evaluation as well as recording of standard electro-oculography and electroretinography were performed in one patient at the onset of visual loss and after 1 year of follow up.

Results-A 61 year old woman presented with visual loss in the right eye. Multiple folds at the level of the internal limiting membrane were seen at the posterior pole in both eyes. Macular oedema was present in the right eye. The visual acuity of the right eye was $6 / 30$ and of the left $6 / 9$. A paracentral scotoma was found in the right eye. Electro-oculographic examination of both eyes gave normal results. Electroretinography (ERG) revealed reduced b-wave and flicker amplitudes in the right eye; these potentials were normal for the left eye. The ON response in the right eye was reduced and delayed; it was normal in the left eye. A further loss of visual function was noted 1 year later in the right eye, but the ophthalmoscopic findings were unchanged. The ERG of the right eye had a negative waveform when dark adapted. Light adapted responses showed an unusual delayed b-wave, broad and delayed $O N$ and $O F F$ responses and a missing flicker response, suggesting a Müller cell dysfunction. Light adapted responses were slightly reduced in the left eye.
\end{abstract}

Conclusions-Electrophysiological data indicate Müller cell dysfunction as a background of functional loss in Müller cell sheen dystrophy. This is in agreement with previously reported histological findings in this disorder.

(Br F Ophthalmol 1998;82:650-654)

A hereditary retinal disorder with alterations of the internal limiting membrane has been characterised recently by Gass and coworkers. ${ }^{12}$ The term "familial internal limiting membranopathy" was initially suggested, ${ }^{1}$ but was subsequently renamed "dominantly inherited Müller cell sheen dystrophy". ${ }^{2}$ There is both histological $^{12}$ and, as demonstrated in this study, electroretinographic evidence for a Müller cell defect in this disorder. We therefore chose to adopt the term "Müller cell sheen dystrophy" in this paper.
Gass and coworkers described a pedigree with four affected patients which suggests an autosomal dominant inheritance pattern. ${ }^{12}$ The ophthalmoscopic features were characterised by multiple folds at the level of the internal limiting membrane at the posterior pole. The folds were smaller outside the temporal vascular arcades and the periphery appeared normal. Macular oedema was present in two patients who developed visual loss after 50 years of age. This clinical appearance is unique and has not been described in any other retinal disorder. The histological examination of two eyes revealed a diffuse thickening and an undulation of the internal limiting membrane. ${ }^{12}$ Another pedigree with similar clinical findings was reported as "autosomal dominant late onset cellophane-like sheen vitreomacular dystrophy" by Dalma et $a l .^{3}$ Ten patients were affected in their pedigree.

Electrophysiological examinations were performed $^{13}$; however, detailed information with regard to recording techniques or normative data was not given. Electro-oculography (EOG) was described as subnormal in one eye and normal in other patients. ${ }^{13}$ In the electroretinogram (ERG) b-wave amplitudes were reduced. ${ }^{1}$

We examined one female patient during the acute stage of visual deterioration and at 1 year follow up. The characteristic ophthalmoscopic findings suggested the diagnosis of Müller cell sheen dystrophy. In this study the results of clinical and electrophysiological evaluation of this disorder are presented.

\section{Patient and methods}

The female patient was first examined at 61 years of age. She had noted acute loss of visual acuity in the right eye 4 weeks previously. The patient had had normal vision before this; further, her general medical history was unremarkable. A basic ophthalmological evaluation, as well as recording of standard EOG and ERG, was performed. The family history could only be partially obtained. The patient's father had no vision problems up to his death at 80 years of age. The patient did not know her mother and she had no brothers or sisters. Her daughter had no visual problems up to the age of 38 years. Our patient had lost contact with her daughter for several years and therefore the daughter was not available for examination.

EOG and ERG were performed as described in detail previously. ${ }^{4}$ The recording techniques were in accordance with the stand- 

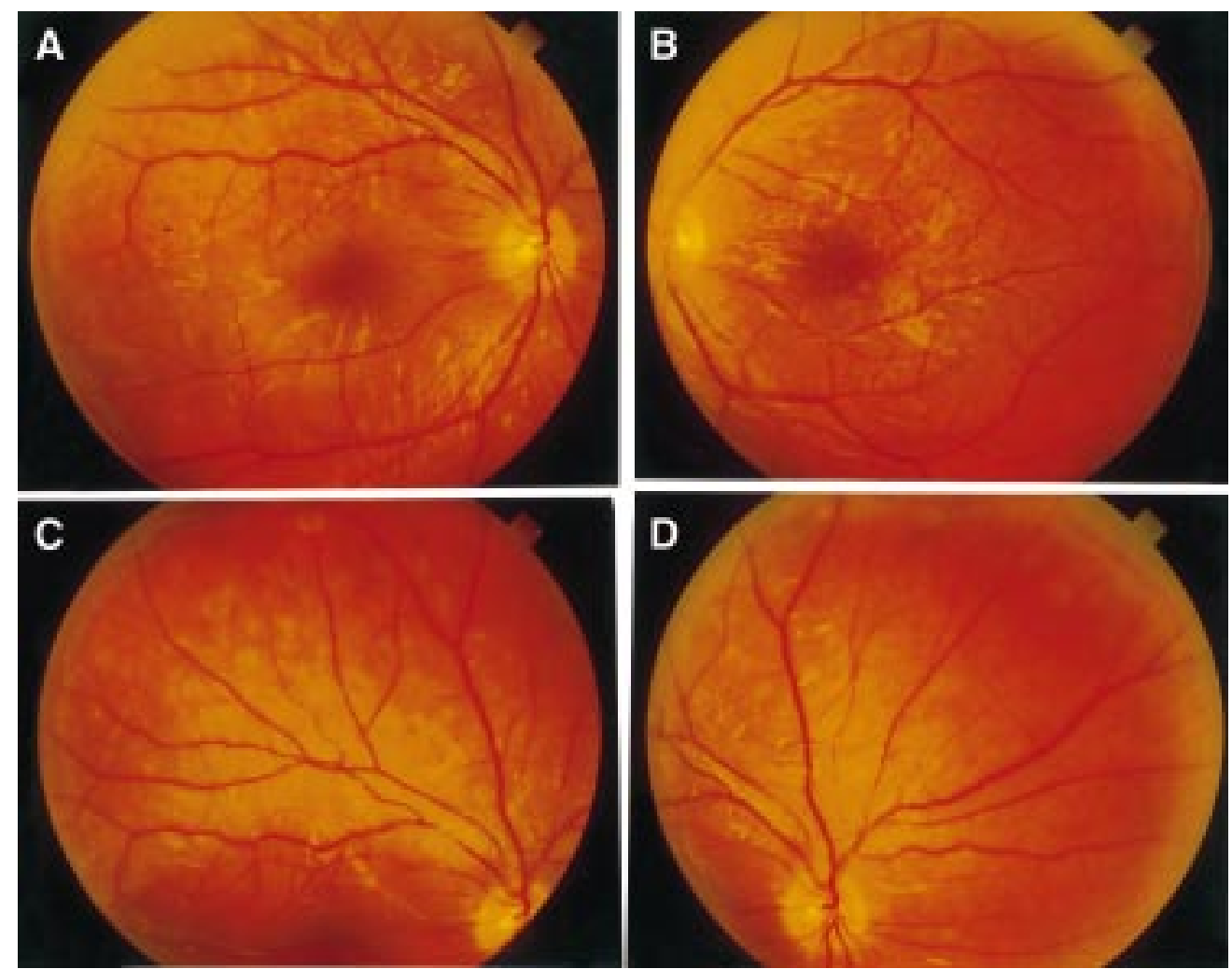

Figure 1 (A) The posterior pole of the right eye shows multiple folds and an oedema in the lower part of the macula. (B) The posterior pole of the left eye shows similar folding, but no oedema. (C) The upper mid periphery of the right eye shows smaller folds in the mid periphery and no folds towards the periphery. (D) The upper nasal quadrant of the right eye shows folds nasally to the optic disc, and no folds towards the periphery. Comparison with (C) shows the variation in visibility of the folds depending on the angle of light as seen in the retina above the optic disc.

ards for clinical EOG and ERG. ${ }^{56}$ Briefly, EOG was performed with maximal dilated pupils $(2.5 \%$ phenylephrine and $0.5 \%$ tropicamide) using a method described by Behrens et al. ${ }^{7}$ The response was described by the ratio between the maximum amplitude and the amplitude before the luminance was increased (light peak versus baseline). Normal ranges for baseline and light peak were defined by calculation of the median values and the $95 \%$ confidence intervals in one eye of 40 probands.

For ERG recording a Nicolet Spirit (Nicolet, Madison, WI, USA) in combination with a Nicolet Ganzfeld was used. Recordings were performed with a Jet contact lens electrode and with maximal dilated pupils. ${ }^{4}$

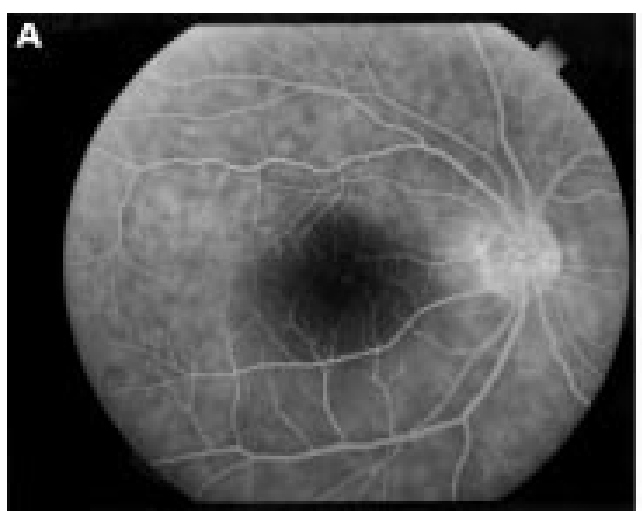

Averaging was not performed. ON and OFF responses were recorded with a LED stimulator (light emitting diode, Roland Consult, Brandenburg, Germany) using either red or green LEDs (3 cd.s $/ \mathrm{m}^{2}$ ) and flashes of long duration $(200 \mathrm{~ms})$ presented on background illumination. Recordings were done after 10 minutes of light adaptation $\left(10 \mathrm{~cd} / \mathrm{m}^{2}\right): 128$ responses were averaged. The normal ranges for ERG and ON and OFF responses were defined by calculation of the median values and the $95 \%$ confidence intervals in one eye of 23 age matched probands.

All examinations were performed in agreement with the Declaration of Helsinki after informed consent was obtained from the patient.

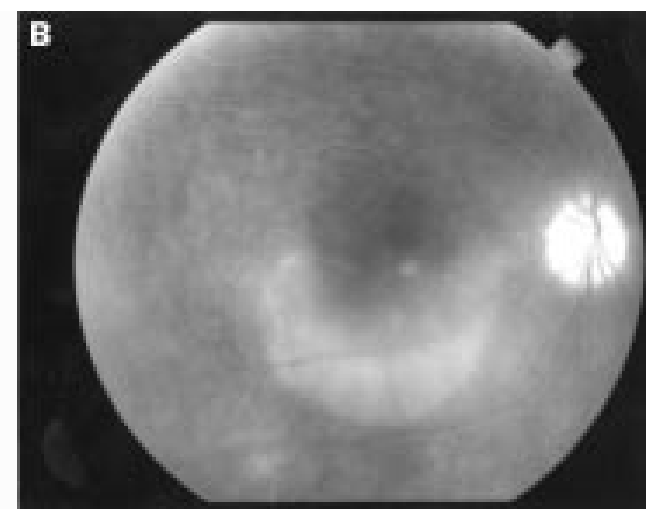

Figure 2 (A) Fluorescein angiography on the right eye reveals normal filling of retinal vessels. In the late venous phase dye leakage begins in a spot near the fovea. (B) In the late phase, dye leakage has stained the lower part of the macula. 
Table 1 ERG amplitudes $(\mu V)$ (reduced amplitudes are underlined)

\begin{tabular}{|c|c|c|c|c|c|c|}
\hline \multirow[b]{2}{*}{ Parameter } & \multirow[b]{2}{*}{ Median } & \multirow[b]{2}{*}{ Lower limit } & \multicolumn{2}{|c|}{ Right eye } & \multicolumn{2}{|c|}{ Left eye } \\
\hline & & & 1996 & 1997 & 1996 & 1997 \\
\hline \multicolumn{7}{|l|}{ Dark adapted: } \\
\hline \multicolumn{7}{|l|}{ Rod: } \\
\hline $\mathrm{b}$ & 445 & 327 & $\underline{122}$ & $\underline{0}$ & 387 & 442 \\
\hline \multicolumn{7}{|l|}{ Rod-cone: } \\
\hline $\mathrm{a}$ & 464 & 376 & 504 & 415 & 505 & 494 \\
\hline $\mathrm{b}$ & 742 & 600 & $\underline{453}$ & $\underline{331}$ & 744 & 744 \\
\hline \multicolumn{7}{|l|}{ Light adapted: } \\
\hline \multicolumn{7}{|l|}{ Single cone: } \\
\hline $\mathrm{a}$ & 103 & 72 & 85 & 100 & 93 & 105 \\
\hline $\mathrm{b}$ & 246 & 200 & $\underline{149}$ & $\underline{127}$ & 271 & $\underline{189}$ \\
\hline Cone flicker: & 241 & 215 & $\underline{99}$ & $\underline{0}$ & 265 & $\underline{200}$ \\
\hline ON response: & 35 & 28 & $\overline{19}$ & $\underline{12}$ & 49 & $\overline{45}$ \\
\hline OFF response: & 32 & 20 & $\overline{29}$ & $\overline{33}$ & 42 & 38 \\
\hline
\end{tabular}

$\mathrm{a}=\mathrm{a}$-wave, $\mathrm{b}=\mathrm{b}$-wave.

\section{Results}

CLINICAL EXAMINATION

At the initial examination (May 1996), the patient complained of hazy vision in the right eye. She had no complaints in the left eye. Visual acuity was right eye $6 / 30(+5.0-0.75$ axis 52) and left eye $6 / 9(+5.75-0.75$ axis 126). Ocular pressure was within the normal range. The anterior segments were unremarkable. Multiple folds of the internal limiting membrane were seen at the posterior pole extending into the mid periphery in both eyes (Fig 1). The outer periphery appeared normal. The degree of folding was similar in both eyes. An additional macular oedema was present in the right eye and was confirmed on fluorescein angiography (Fig 2). Vascular leakage was not present outside of the macula.

Goldmann visual fields showed normal outer isoptres. A small paracentral scotoma was present in the right eye. Colour vision testing with the desaturated Panel D15 test revealed mild (left eye) to severe (right eye) errors without a typical axis of confusion.

At the follow up examination (April 1997), the patient complained of further visual loss in the right eye. Visual acuity in the right eye was reduced to 6/120. It was unchanged in the left eye. The ophthalmoscopic findings with respect to the location and severity of the folds were unchanged in both eyes. A macular oedema was still present in the right eye. The patient did not agree to repeat fluorescein angiography. Goldmann visual fields showed a slight increase of the paracentral scotomas and a loss for small targets in the right eye. Visual fields remained normal in the left eye.

ELECTROPHYSIOLOGICAL EXAMINATION

An EOG was recorded once at the time of the first examination. Baseline values and light peak were normal in both eyes (baseline: right

Table 2 ERG implicit times (ms) (delayed implicit times are underlined)

\begin{tabular}{|c|c|c|c|c|c|c|}
\hline \multirow[b]{2}{*}{ Variable } & \multirow[b]{2}{*}{ Median } & \multirow[b]{2}{*}{ Upper limit } & \multicolumn{2}{|c|}{ Right eye } & \multicolumn{2}{|c|}{ Left eye } \\
\hline & & & 1996 & 1997 & 1996 & 1997 \\
\hline Single cone & 32.0 & 34.8 & 34.0 & nd & 32.0 & 30.8 \\
\hline Cone flicker & 29.3 & 31.5 & 32.0 & $\mathrm{~nm}$ & 28.0 & 28.0 \\
\hline ON response & 32.0 & 36.0 & 38.0 & 50.0 & 33.0 & 34.0 \\
\hline OFF response & 22.0 & 24.0 & $\overline{26.0}$ & $\overline{42.0}$ & 23.0 & 24.0 \\
\hline
\end{tabular}

nd=not determinable, because no distinct $b$-wave peak was present. $\mathrm{nm}=$ not measurable owing to missing response.
A

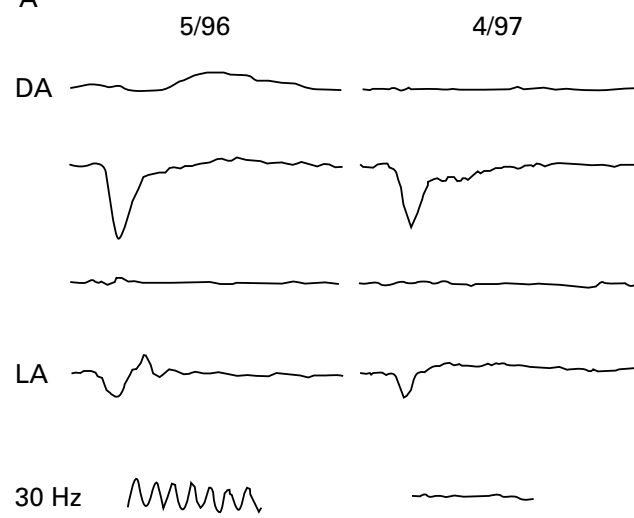

B

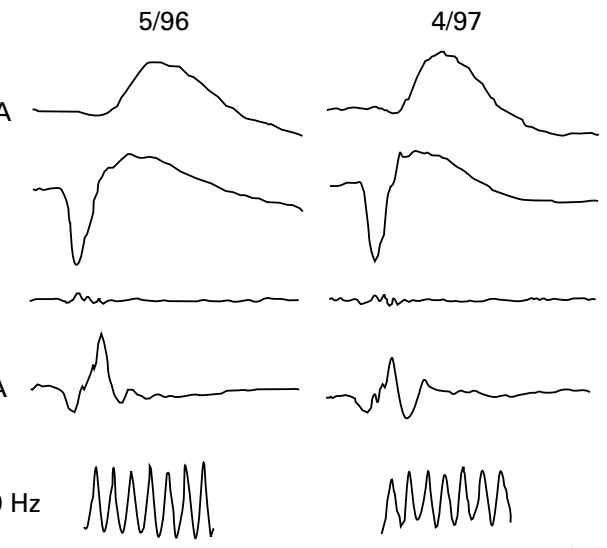

Figure 3 (A) ERG in response to white stimuli of the right eye of our patient at the initial (5/96) and final examination (4/97). DA indicates responses at dark adaptation at low stimulus intensity (rod response, first row) and maximum stimulus intensity (mixed rod-cone response, second row). The next row shows the dark adapted oscillatory potentials. LA indicates single flash responses at light adaptation (cone response). $30 \mathrm{~Hz}$ indicates the cone flicker response. Vertebral calibration mark indicates 200 $\mu V$ for $D A$ recordings and $100 \mu V$ for $L A$ and flicker recordings. Horizontal calibration is $20 \mathrm{~ms}$ for single flash recordings and $50 \mathrm{~ms}$ for flicker recordings. (B) ERG in response to white stimuli of the left eye of our patient at the initial and final examination. The responses of the left eye at the initial examination are normal. For further explanation see $(A)$.

eye $0.46 \mathrm{mV}$, left eye: $0.48 \mathrm{mV}$; normal median: $0.47 \mathrm{mV}$, lower normal range: 0.25 $\mathrm{mV}$; light peak: right eye: $188 \%$, left eye: $214 \%$; normal median: $186 \%$, lower normal range: $160 \%)$.

ERGs were recorded at the first visit and at the follow up examination. The values for amplitudes and implicit times are given in Tables 1 and 2; the recordings are shown in Figures 3 and 4 .

Right eye

At the first examination, recording at dark adaptation revealed normal a-wave amplitudes and reduced b-wave amplitudes (Fig 3). At maximum stimulus intensity, the b-wave amplitude was reduced to $61 \%$ of normal median. The ERG response had the configuration of a negative ERG (b-wave amplitude smaller than a-wave amplitude). At light adaptation, the a-wave amplitude, was in the lower normal 

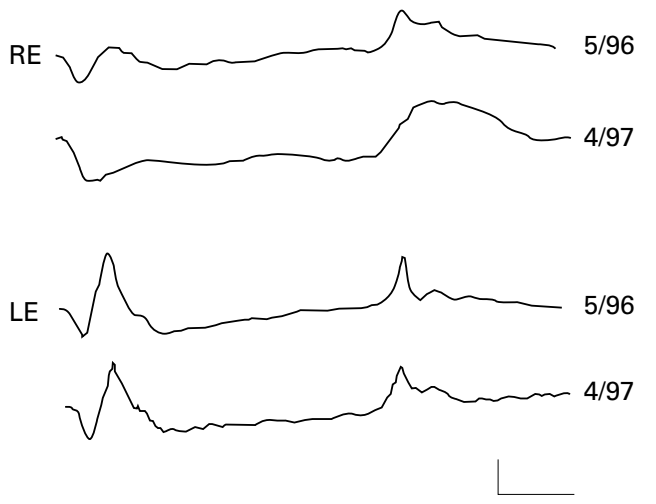

Figure $4 O N$ and $O F F$ responses to red LED stimuli of $200 \mathrm{~ms}$ duration of both eyes of our patient at the initial (5/96) and final examination (4/97). The responses of the left eye at the initial examination are normal. Vertical axis indicates $50 \mu \mathrm{V}$ and horizontal axis calibration of $50 \mathrm{~ms}$.

range. The b-wave amplitude was reduced to $60 \%$ of normal median. The b-wave implicit time was $2 \mathrm{~ms}$ longer compared with the left eye, but it was still within the upper normal range. The $30 \mathrm{~Hz}$ flicker amplitude was reduced to $40 \%$ of normal median and the implicit time was slightly prolonged. The ON response amplitude was reduced to $54 \%$ and the OFF response amplitude was normal (Fig 4). The ON and OFF response implicit times were slightly delayed.

Recording at dark adaptation 1 year later demonstrated a-wave amplitudes in the lower normal range. B-wave amplitudes were further reduced at all stimulus intensities (Fig 3). At maximum stimulus intensity, the b-wave amplitude was reduced to $45 \%$ of normal median. The ERG response had the configuration of a negative ERG. At light adaptation, the a-wave amplitude was normal and the b-wave amplitude was reduced to $50 \%$ of normal median. The configuration of the b-wave showed a delayed return to baseline; the implicit time was, therefore, not determinable. The $30 \mathrm{~Hz}$ flicker response was no longer recordable. A remarkable change had appeared in the $\mathrm{ON}$ and $\mathrm{OFF}$ responses (Fig 4). The onset of the $\mathrm{ON}$ and $\mathrm{OFF}$ responses was normal; however, the waveform of both was broader than normal and the return to baseline was delayed. The ON response amplitude was further reduced; the OFF response amplitude remained normal. The implicit times of both $\mathrm{ON}$ and $\mathrm{OFF}$ responses were markedly delayed.

Left eye

At the first examination, the ERG recording revealed normal a- and b-wave amplitudes and implicit times at all stimulus conditions. The $30 \mathrm{~Hz}$ flicker response as well as $\mathrm{ON}$ and $\mathrm{OFF}$ responses were normal in amplitude and timing (Figs 3 and 4). One year later, dark adapted recordings were unchanged. At light adaptation, the a-wave amplitude remained normal. The b-wave amplitude, however, was subnormal (75\% of normal median) and was about $30 \%$ lower compared with the previous examination. The $30 \mathrm{~Hz}$ flicker amplitude was reduced to a borderline level, but was $25 \%$ lower compared with the previous recording.
$\mathrm{ON}$ and $\mathrm{OFF}$ responses were slightly reduced compared with the previous examination, but the amplitudes were still within the normal range and the implicit times remained unchanged.

\section{Discussion}

The ophthalmoscopic findings in our patient resemble the findings described previously. ${ }^{1-3}$ Based on these characteristic features and a similar age of onset, we diagnosed Müller cell sheen dystrophy, although an autosomal dominant inheritance could not be demonstrated.

The functional disturbance in the left eye of our patient was mild despite the marked folds at the posterior pole. Indeed, she had not noted any vision problems in the left eye at all. Slowly progressive functional loss was, however, obvious in the light adapted ERG recordings after 1 year of follow up. Functional loss was, in contrast, more severe in the right eye. The most interesting findings were seen in the ERG; the negative waveform at dark adaptation, the delayed implicit times in light adapted responses, and the loss of the $30 \mathrm{~Hz}$ flicker response. Based on functional and ophthalmoscopic findings other retinal disorders associated with a negative waveform in the ERG (for example, congenital stationary night blindness, $\mathrm{X}$ linked retinoschisis, subtypes of retinitis pigmentosa or cone dystrophies, melanoma associated retinopathy, and severe retinal vascular disorders ${ }^{4-14}$ ) could be excluded in our patient. Reduced ERG amplitudes and a negative waveform have been described in other patients with Müller cell sheen dystrophy. ${ }^{13}$

The normal a-wave amplitudes as well as the normal EOG in both eyes of our patient indicate normal function of the photoreceptorpigment epithelium complex. Retinal bipolar cells and Müller cells are predominantly involved in the generation of the b-wave of the clinical ERG. ${ }^{15-20}$ The reduced b-wave and flicker amplitudes at both examinations in the right eye and at the last examination in the left eye suggest a functional defect either in bipolar cells or Müller cells. The $\mathrm{ON}$ response reflects activity of the depolarising bipolar cells, and the OFF response activity of the hyperpolarising bipolar cells. ${ }^{16}$ At the last follow up examination in the right eye of our patient, the onsets of $\mathrm{ON}$ and OFF responses were normal. Both responses showed a broader waveform with markedly delayed implicit times. This broader waveform of both responses could be explained by a delayed uptake of $\mathrm{K}^{+}$ions in the Müller cells. A similar delay of implicit time of $\mathrm{ON}$ and $\mathrm{OFF}$ responses is in accordance with normal bipolar cell function in combination with a Müller cell defect. Therefore, the negative ERG at dark adaptation and the delayed implicit times at light adaptation in the right eye of our patient most likely indicate Müller cell dysfunction. The delayed implicit times of $\mathrm{ON}$ and $\mathrm{OFF}$ responses have two interesting consequences with regard to the waveform of the light adapted ERG. The b-wave of the single cone response is broader than usual and the return to baseline is delayed. The complete loss of the $30 \mathrm{~Hz}$ flicker response within 1 year can 
be explained by the increased delay of both $\mathrm{ON}$ and OFF responses. The potentials of both depolarising and hyperpolarising bipolar cells and, to a lesser degree, the cone photoreceptor potentials have been recently demonstrated to constitute the $30 \mathrm{~Hz}$ flicker response. ${ }^{21}$ The delayed timing of both bipolar cell potentials apparently alters the summation for the flicker response, resulting in the elimination of a recordable flicker response.

The basement membranes of the Müller cells form the main part of the internal limiting membrane. ${ }^{22}{ }^{23}$ Histologically, a diffuse thickening and an undulation of the internal limiting membrane with small inner retinal schisis cavities have been demonstrated in Müller cell sheen dystrophy. ${ }^{12}$ We can speculate that the primary defect in this disorder is within the Müller cells. During the early stage of the disease, Müller cells may produce a material which has yet to be characterised in nature and which accumulates in the internal limiting membrane. This material deposition induces thickening of the membrane and its subsequent folding. At this stage, the membrane properties of the Müller cells show normal function as far as they contribute to the generation of the b-wave of the ERG. This is demonstrated in the left eye of our patient at the first examination. At a later stage, the increased accumulation of material leads to disruption of the contact between the internal limiting membrane and the Müller cells and, consequently, inner retinal schisis cavities develop. ${ }^{12}$ The functional result includes the deterioration of the b-wave amplitude and the delayed implicit times, as seen in the right eye of our patient. The widespread increased retinal vascular permeability in the late stage of the disease, as observed in some patients, ${ }^{12}$ may be due to secondary damage to retinal capillaries, which are surrounded by a Müller cell basement membrane. ${ }^{1{ }^{24}}$ It is reasonable to assume that the most severe folding occurs at the posterior pole since the internal limiting membrane is thicker at the posterior pole than in the periphery. ${ }^{23}{ }^{25}$ The onset of clinical signs at 50 years of age or later may be accounted for by the normal thickening of the internal limiting membrane with age. ${ }^{23} 26$

Treatment has not proved to be of benefit for patients with Müller cell sheen dystrophy. Vitrectomy performed in three eyes did not improve their visual function. ${ }^{1}$ We initiated a treatment with systemic acetazolamide and local flurbiprofen because this has been proved to be helpful in some cases of macular oedema from other causes. ${ }^{47}$ A positive effect of this treatment, however, could not be observed in our patient.
This study was partially supported by the Deutsche Forschungsgemeinschaft, Germany (U Kellner, grant DFG-Ke442/ ungsgen.

1 Polk TD, Gass JDM, Green WR, et al. Familial internal limiting membrane dystrophy: a new sheen retinal dystrophy. Arch Ophthalmol 1997;115:878-85.

2 Gass JDM. Stereoscopic atlas of macular diseases diagnosis and treatment. 4th ed. St Louis: Mosby, 1997:340-2.

3 Dalma A, Dalma-Weiszhausz J, Jimenez-Sierra JM. Autosomal dominant late onset celophane-like sheen vitreomacular dystrophy:a new entity. Invest Ophthalmol Vis Sci 1991;32:1228.

4 Kellner U, Bornfeld N, Foerster MH. Severe course of cutaneous melanoma associated paraneoplastic retinopathy. $\mathrm{Br}$ neous melanoma associated pa
f Ophthalmol 1995;79:746-52.

5 Marmor MF, Zrenner E. Standard for clinical electroocuMarmor MF, Zrenner E. Standard for clinical
lography. Arch Ophthalmol 1993;111:601-4.

6 Marmor MF, Arden GB, Nilsson SEG, et al. Standard for clinical electroretinography. Arch Ophthalmol 1989;107: 816-9.

7 Behrens F, Foerster MH, Petsch J, et al. A new apparatus measuring the clinical electrooculogram (DC-EOG) automatically. Ger f Ophthalmol 1993;2:382.

8 Miyake Y, Yagasaki K, Horiguchi M, et al. Congenital stationary night blindness with negative electroretinogram. A new classification. Arch Ophthalmol 1986;104:1013-20.

Kellner U, Brümmer S, Foerster MH, et al. X-linked 1990;228:432-7.

10 Cideciyan AV, Jacobson SG. Negative electroretinograms in retinitis pigmentosa. Invest Ophthalmol Vis Sci 1993;34: 3253-63.

11 Kellner U, Foerster MH. Cone dystrophies with negative photopic electroretinogram. Br F Ophthalmol 1993;77:404-

12 Miyake Y, Shiroyama N, Horiguchi M, et al. Bull's-eye maculopathy and negative electroretinogram. Retina 1989; 9:210-5.

13 Alexander KR, Fishman GA, Peachey NS, et al. 'On' response defect in paraneoplastic night blindness with cutaneous malignant melanoma. Invest Ophthalmol Vis Sci 1992;33:477-83.

14 Johnson MA. b/a-wave amplitude ratios in retinal vascular disease. In: Heckenlively JR, Arden GB, eds. Principles and practice of clinical elelctrophysiology of vision. St Louis: Mosby, 1991:531-6.

15 Miller RF, Dowling JE. Intracellular responses of the Müller (glial) cells of the mudpuppy retina: their relation to b-wave (glial) cells of the mudpuppy retina: their relation to b-wave

16 Sieving PA, Murayama K, Naarendorp F. Push-pull model of the primate photopic electroretinogram:A role for hyperpolarizing neurons in shaping the b-wave. Vis Neurosci 1994;11:519-32.

17 Stockton RA, Slaughter MM. B-wave of the electroretinogram. A reflection of ON bipolar cell activity. $\mathcal{F}$ Gen Physiol 1989;93:101-22.

18 Gurevich L, Slaughter MM. Comparison of the waveforms of the ON bipolar neuron and the b-wave of the electroretinogram. Vis Res 1993;33:2431-5.

19 Tian N, Slaughter MM. Correlation of dynamic responses in the ON bipolar neuron and the b-wave of the electroretinogram. Vis Res 1995;35:1359-64.

20 Newman EA, Odette LL. Model of electroretinogram b-wave generation: a test of the $\mathrm{K}+$ hypothesis. $f$ Dewrophysiol 1984;51:164.

21 Kim S, Bush RA, Sieving PA. Monkey photopic flicker ERG to sine and square wave stimuli:component identification using drug isolation. Invest Ophthalmol Vis Sci 1997;38: S885.

22 Reichenbach A, Robinson SR. The involvement of Müller cells in the outer retina. In: Djamgoz MBA, Archer SN, Vallerga S, eds. Neurobiology and clinical aspects of the outer retina. London: Chapman \& Hall, 1995:395-416.

23 Green WR. Vitreoretinal juncture. In: Ryan SJ, ed. Retina. Vol 3. 2nd ed. St Louis: Mosby, 1994:1871-4.

24 Carlson EC. Human retinal capillary basement membrane leaflets are morphologically distinct: a correlated TEM and SEM analysis. Exp Eye Res 1989;49:967-81.

25 Foos RY. Vitreoretinal juncture:topographical variations. Invest Ophthalmol 1972;11:801-8.

26 Rentsch FJ, van der Zypen E. Altersbedingte Veränderungen der sog. Membrana limitans interna des Ziliarkörpers menschlichen Auge. In: Bredt H, Rohen TW, eds. Altern und Entwicklung. Stuttgart: Schattauer, 1971:70-94.

27 Fishman GA, Gilbert LD, Fiscella RG, et al. Acetazolamide for treatment of chronic macular edema in retinitis pigmentosa. Arch Ophthalmol 1989;107:1445-52 